\title{
Assessment of direct analgesic effect of duloxetine for chronic low back pain: post hoc path analysis of double-blind, placebo-controlled studies
}

This article was published in the following Dove Press journal: Journal of Pain Research

I June 2017

Number of times this article has been viewed

\author{
Hiroyuki Enomoto' \\ Shinji Fujikoshi ${ }^{2}$ \\ Jumpei Funai ${ }^{3}$ \\ Nao Sasaki ${ }^{4}$ \\ Michael H Ossipov ${ }^{5}$ \\ Toshinaga Tsuji ${ }^{6}$ \\ Levent Alev ${ }^{7}$ \\ Takahiro Ushida ${ }^{8}$
}

'Medical Science, Eli Lilly Japan K.K., Tokyo, ${ }^{2}$ Statistical Science, ${ }^{3}$ Science Communications, ${ }^{4}$ Medical Science, Eli Lilly Japan K.K., Kobe, Japan; ${ }^{5}$ Clinical Division, inVentiv Health, LLC, Blue Bell, PA, USA; ${ }^{\circ}$ Medical Affairs Department, Shionogi \& Co., Ltd., Osaka, Japan; ${ }^{7}$ Medical Department, Lilly Turkey, Istanbul, Turkey; ${ }^{8}$ Multidisciplinary Pain Center, Aichi Medical University, Nagakute, Aichi, Japan
Correspondence: Hiroyuki Enomoto Medical Science, Eli Lilly Japan K.K., 4-I5-I-I3F, Akasaka, Minato-ku, Tokyo 107-0052, Japan

Tel +8I 355749143

Fax +81355749979

Email enomoto_hiroyuki@lilly.com
Background: Comorbid depression and depressive symptoms are common in patients with chronic low back pain (CLBP). Duloxetine is clinically effective in major depressive disorder and several chronic pain states, including CLBP. The objective of this post hoc meta-analysis was to assess direct and indirect analgesic efficacy of duloxetine for patients with CLBP in previous clinical trials.

Methods: Post hoc path analyses were conducted of 3 randomized, double-blind, clinical studies of patients receiving duloxetine or placebo for CLBP. The primary outcome measure for pain was the Brief Pain Inventory, average pain score. A secondary outcome measure, the Beck Depression Inventory-II, was used for depressive symptoms. The changes in score from baseline to endpoint were determined for each index. Path analyses were employed to calculate the proportion of analgesia that may be attributed to a direct effect of duloxetine on pain.

Results: A total of 851 patients ( 400 duloxetine and 451 placebo) were included in this analysis. Duloxetine significantly improved pain scores compared with placebo $(p<0.001)$. It also significantly improved depressive scores compared with placebo $(p=0.015)$. Path analyses showed that $91.1 \%$ of the analgesic effect of duloxetine could be attributed to a direct analgesic effect, and $8.9 \%$ to its antidepressant effect. Similar results were obtained when data were evaluated at weeks 4 and 7, and when patients were randomized to subgroups based on baseline pain scores, baseline depressive symptoms scores, and gender.

Conclusion: Duloxetine significantly improved pain in patients with CLBP. Path analyses results suggest that duloxetine produced analgesia mainly through mechanisms directly impacting pain modulation rather than lifting depressive symptoms. This effect was consistent across all subgroups tested.

Keywords: duloxetine, direct analgesic effect, chronic low back pain, post hoc path analysis, double-blind, placebo-controlled studies

\section{Introduction}

Chronic low back pain (CLBP) is a significant global public health concern, and the major cause of disability. ${ }^{1}$ CLBP is a leading cause of physical disability in individuals aged $<45$ years. $^{2}$ In Japan, the point prevalence of CLBP ranges between $20 \%$ and $25 \%,{ }^{1}$ and the lifetime prevalence was found to be $83 \%$, which is comparable to that of countries such as Canada, Germany, and Australia. ${ }^{3}$

CLBP is highly disabling and is the leading cause of work days lost. ${ }^{3-6}$ In addition to its physical impact, various studies have found that individuals with CLBP also have significantly greater levels of depressive symptoms, anxiety, and sleep impairment compared with matched controls without CLBP. ${ }^{7-10}$ For most (>90\%) patients 
with anxiety disorders or substance abuse, the syndromes preceded the onset of CLBP, whereas for $46 \%$ of those with comorbid depressive symptoms, the depressive symptoms appeared after the onset of CLBP. ${ }^{10}$ There appears to be a reciprocal association between the development of depressive symptoms and back pain, as depression tends to exacerbate pain in CLBP patients, and increased pain is associated with greater risk of depression. ${ }^{8,9}$

The etiology of CLBP remains largely unknown, thereby presenting a challenge in optimizing treatment. ${ }^{11}$ Although $\sim 15 \%$ of cases may be attributed to a specific cause, such as osteoporotic fracture, neoplasm, or infection, $85 \%$ of CLBP cases are idiopathic, with no clear cause. ${ }^{2}$ Factors that are associated with back pain include obesity, arthritis, increase in age, poor posture, poor physical condition, and psychologic factors such as stress, anxiety, depression, and smoking, but these factors are not highly correlative and do not predict patients at risk of developing CLBP. ${ }^{2,12,13}$ Growing evidence indicates that CLBP, like many other chronic pain conditions, is largely driven by central sensitization, neuronal hyperactivity, and dysfunction of endogenous pain modulatory systems. ${ }^{12,14-18}$ In one recent study, it was shown that patients with CLBP had significantly decreased conditioned pain modulation compared to normal, healthy volunteers. ${ }^{15}$ Taken together, these studies indicate that dysfunctional pain modulation and enhanced excitability are at least partially responsible for the development and the persistence of chronic pain regardless of the underlying etiology.

Duloxetine, a potent and selective serotonin and norepinephrine reuptake inhibitor, is believed to exert a direct analgesic effect through modulation of endogenous descending inhibitory pain modulatory systems. ${ }^{19,20}$ In addition to being effective in major depressive disorder (MDD), duloxetine has also been demonstrated to be efficacious in the treatment of CLBP as well as other chronic pain conditions such as diabetic neuropathy, fibromyalgia, and osteoarthritis of the knee. Recently, on the strength of 4 randomized clinical trials, duloxetine has been approved for the treatment of CLBP in the US and Japan. ${ }^{6,11,17,21}$ It is known that chronic pain is often related to depressive symptoms. However, it is unknown whether duloxetine has an independent analgesic effect. That is the reason why path analysis was conducted in CLBP patients without MDD. Previously, the results from double-blind, placebo-controlled studies reported that duloxetine significantly reduced painful physical symptoms and improved depression in patients with MDD. ${ }^{22,23}$ Also, early reduction in painful physical symptoms was associated with improvements in long-term depression outcomes in patients treated with duloxetine.
Path analysis is a means to decompose correlations in order to find what correlations may exist among variables in producing an effect.

In the present investigation, path analyses were used to evaluate the direct (ie, analgesic) and indirect (via antidepressant effect) effects of duloxetine in relieving CLBP.

\section{Methods}

\section{Data sources and study selection}

The Eli Lilly clinical trial database was searched for randomized, double-blind, placebo-controlled, parallel-group clinical trials with patients receiving duloxetine or placebo who had CLBP. Importantly, MDD was an exclusion criterion for all of the CLBP studies. Consequently, none of the patients included in this study was diagnosed with MDD. A total of 4 studies, HMEN (NCT00424593), ${ }^{17}$ HMEO (NCT00408876), ${ }^{11}$ HMGC (NCT00767806), ${ }^{6}$ and HMGY (NCT01855919), ${ }^{21}$ were identified. Studies HMGC, HMEO, and HMEN were conducted independently over the time period from December 2006 through November 2009 and HMGY was conducted from May 2013 through July 2014. The details of the study designs and results have been published previously., ${ }^{6,11,17,21}$ One of these studies, HMGC, was not included in the present analyses because the secondary outcome measure used to determine depressive symptoms was the Profile of Mood States - Brief Form, ${ }^{6}$ whereas depressive symptoms were determined by the Beck Depression Inventory-II (BDI-II) in the remaining 3 studies. ${ }^{11,17,21}$

The protocols were approved by the appropriate ethical review boards for each of the study centers. Subjects gave written informed consent before participating and were fully informed of the procedures involved in the studies. The studies were all conducted in accordance with the regulatory standards in each country and conformed to the standards dictated by Good Clinical Practice, the Declaration of Helsinki (2008), and all applicable local regulations. The studies were registered at ClinicalTrials.gov.

\section{Study population}

A post hoc, individual, patient-level meta-analysis of data was conducted from studies HMEN, HMEO, and HMGY to better understand the direct analgesic effect of duloxetine in patients with CLBP. The common inclusion criteria were male or female patients $\geq 18$ years of age ( 20 years for HMGY), low back pain present on most days for 6 months, and a pain score of $\geq 4$ on the Brief Pain Inventory (BPI). Study HMGY included an inclusion criterion of nonsteroidal antiinflammatory drug use for CLBP for an average of $>14$ days 
per month in the past 3 months and for $>14$ days in the month prior to screening.

Patients with evidence of radicular compression or other vertebrae-related disorder (eg, spinal stenosis, spondylolisthesis), history of $>1$ low back surgery or surgery within the past 12 months, or invasive treatment for CLBP within the past month were excluded. Importantly, the studies excluded patients with MDD as determined by the depression module of the Mini International Neuropsychiatric Interview. Specific inclusion and exclusion criteria are described in the primary publications. ${ }^{11,17,21}$ Patients were included in this post hoc analysis if they were randomized to duloxetine $60 \mathrm{mg}$ or placebo. However, those patients who were randomized to $60 \mathrm{mg}$ and escalated up to $120 \mathrm{mg}$ at week 7 in HMEN were excluded from the analysis. ${ }^{17}$

\section{Assessments}

Pain was assessed with the BPI 24-hour average pain scores, which measures average pain experienced over the previous 24 hours on a scale from 0 (no pain) to 10 (pain as bad as you can imagine). Improvement in pain is indicated by a reduction in the BPI score from the baseline BPI average score. Depressive symptoms were assessed with the BDI-II, which is a 21 -item questionnaire completed by the patient in order to assess characteristics of depressive symptoms. The BDI-II consists of 21 questions that are each scored from 0 to 3. Consequently, the results can range from 0 to the highest possible total of $63 .{ }^{22} \mathrm{~A}$ score of $0-10$ is considered normal, 11-16 indicates a mild mood disturbance, and a score of $\geq 31$ indicates severe depression. ${ }^{24}$
Path analysis was used to confirm that duloxetine has a direct effect, distinct from its antidepressant activity, in improving pain in patients with CLBP. Path analysis is related to multiple regression methods and is a means to decompose correlations in order to determine the correlations among variables on an effect. ${ }^{25,26}$ In this investigation, path analysis was performed to determine the direct effect of duloxetine treatment on chronic pain and the indirect effects on chronic pain due to improvement in depressive symptoms over time. The 2 regression models employed in the path analyses are represented as:

$$
\begin{aligned}
& X_{3}=\alpha_{0}+\alpha_{1} X_{1}+\alpha_{2} X_{2}+\alpha_{3} Z_{1}+\alpha_{4} Z_{2} \\
& \quad \text { and } \\
& X_{2}=\beta_{0}+\beta_{1} X_{1}+\beta_{2} Z_{1}+\beta_{3} Z_{2}
\end{aligned}
$$

in which $\mathrm{X}_{3}$ is the change from baseline in BPI average pain score, $\mathrm{X}_{2}$ is the change from baseline in BDI-II total score, $X_{1}$ is the treatment, $Z_{1}$ is the baseline BDI-II total score, and $\mathrm{Z}_{2}$ is the baseline BPI average pain score. In each model, study was also included as a covariate. The direct and indirect effects of duloxetine were estimated by $\alpha_{1}$ and $\alpha_{2} \times \beta_{1}$, respectively, Figure 1).

Path analyses were performed with improvement in BPI and BDI-II for the entire population of the 3 studies combined at the endpoint. In addition, time-course analyses were performed with data from studies HMEN and HMEO at weeks 4,7 , and 13 to determine if there is a time course in the relative contributions of the direct effect and indirect effects of duloxetine on pain. In addition, subgroups formed

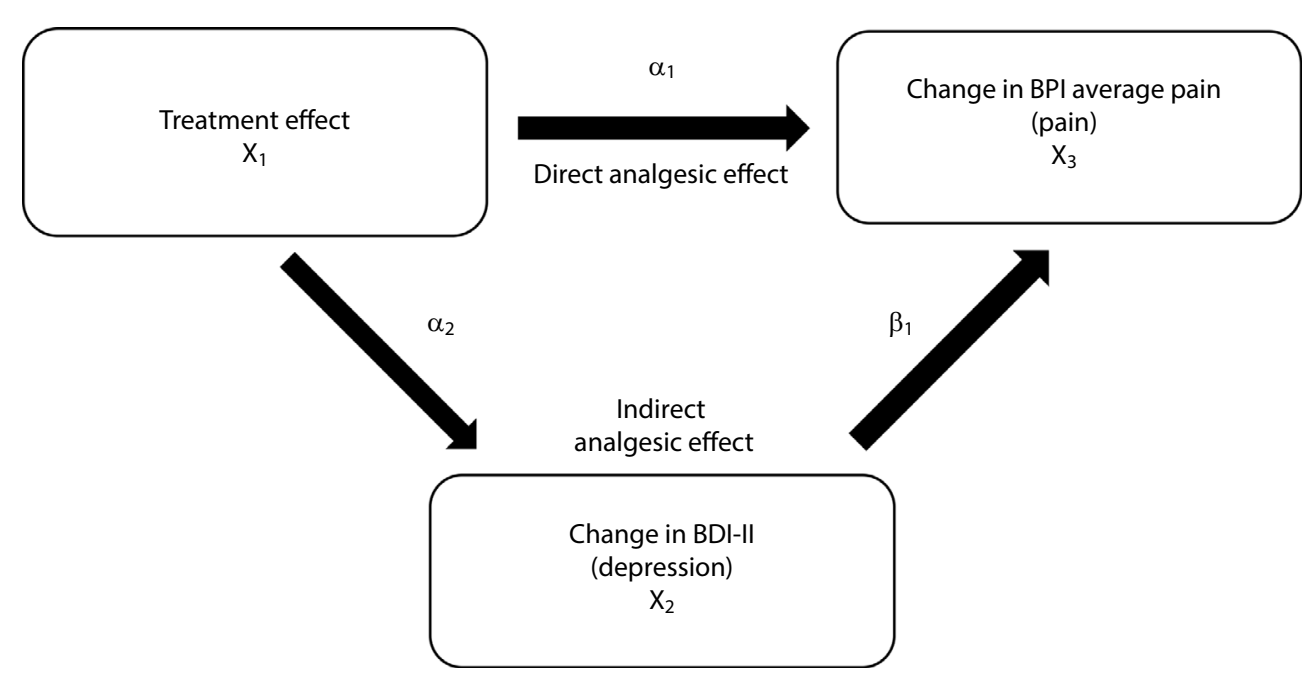

Figure I Path analysis diagram that illustrates direct (BPI) and indirect (BDI-II) effects of treatment on improvement in pain symptoms. The direct analgesic effect is represented by $\alpha_{1}$, and the indirect analgesic effect is represented by $\alpha_{2}$ (improvement of depression) and $\beta_{1}$ (improvement of pain due to improvement of depression). Abbreviations: BDI-II, Beck Depression Inventory-II; BPI, Brief Pain Inventory. 
from the 3 studies were analyzed to determine whether path analyses would reveal changes in the direct and indirect components of duloxetine on pain relief in patients with baseline BPI $<6$ versus $\mathrm{BPI} \geq 6$, baseline $\mathrm{BDI}-\mathrm{II}<6$ versus $\mathrm{BDI}-\mathrm{II} \geq 6$, and in males versus females.

\section{Results}

\section{Patient demographics}

The data from all randomized patients in all 3 studies were examined, and patients who had a recorded baseline value and at least one post-baseline value for both BPI and BDI-II were included in the analysis. Consequently, a total of 851 patients (duloxetine $60 \mathrm{mg}, \mathrm{n}=400$; placebo, $\mathrm{n}=451$ ) were included in this analysis (Table 1, Figure 2). The baseline demographics and clinical characteristics were similar between the two groups. The baseline BPI average pain score (5.5 and 5.6, respectively, for duloxetine and placebo) indicated moderate pain levels at study enrollment. Patients showed minimal depressive symptoms, as demonstrated by low scores on the BDI-II scale in the Japanese and US trials, which is consistent with the exclusion of patients with MDD (Table 1).

\section{Patient disposition}

Completion rates were similar (>80\%) in the duloxetine and placebo groups (Figure 2). A higher percentage of patients in the placebo group discontinued because of lack of efficacy and patient decision compared with the duloxetine group. On the contrary, a higher percentage of patients in the duloxetine group discontinued because of an adverse event compared with the placebo group.

\section{Efficacy of duloxetine treatment at endpoint}

Pain scores determined by the BPI were reduced by duloxetine over the course of the study (Figure 3). Patients who were treated with duloxetine showed significantly $(p<0.001)$ greater reductions in BPI average pain than did those who received placebo (Figure 3A). Duloxetine also produced significantly greater reductions in depressive symptoms over the course of the study compared with the placebo group (Figure 3B). Path analyses revealed that $91.1 \%$ of the improvement in pain symptoms was due to a direct effect of duloxetine, and $8.9 \%$ due to an indirect effect through an improvement in depressive symptoms (Figure 3C).

\section{Time course of duloxetine-induced pain relief}

The data from patients in studies HMEN and HMEO were pooled in order to perform path analyses over time. Study HMGY was not included because the BDI-II data were collected only at baseline and the endpoint of the study. The baseline BPI averages for the duloxetine and placebo groups were (mean \pm standard deviation [SD]) $5.5 \pm 1.45$ and 5.6 \pm 1.47 , respectively. Duloxetine treatment resulted in progressive reductions in the BPI scores over time that were significantly greater than those of the placebo-treated group (Figure 4A). The direct effect of duloxetine for analgesia was slightly reduced over time but remained $>80 \%$ (Figure 4C).

Table I Baseline demographics and clinical characteristics

\begin{tabular}{|c|c|c|c|}
\hline Baseline characteristics & Duloxetine $(n=400)$ & Placebo $(n=45 I)$ & Total $(\mathbf{N}=85 \mathrm{I})$ \\
\hline \multicolumn{4}{|l|}{ Gender } \\
\hline Female, n (\%) & $218(54.5)$ & $254(56.3)$ & $472(55.5)$ \\
\hline Male, n (\%) & $182(45.5)$ & $197(43.7)$ & $379(44.5)$ \\
\hline \multicolumn{4}{|l|}{ Age (years) } \\
\hline Mean (SD) & $56.8(14.4)$ & $55.1(13.8)$ & $55.9(14.1)$ \\
\hline Median (range) & $58.0(19.7-85.4)$ & $56.0(21.2-90.9)$ & $57.0(19.7-90.9)$ \\
\hline \multicolumn{4}{|l|}{ Duration of CLBP (years) } \\
\hline Mean (SD) & $10.0(10.2)$ & $10.0(9.6)$ & $10.0(9.9)$ \\
\hline Median (range) & $6.5(0.5-54.0)$ & $7.0(0.4-64.2)$ & $6.9(0.4-64.2)$ \\
\hline \multicolumn{4}{|c|}{ Baseline BPI average pain score } \\
\hline Mean (SD) & $5.5(1.5)$ & $5.6(1.5)$ & $5.5(1.5)$ \\
\hline Median (range) & $5.0(2.0-10.0)$ & $5.0(1.0-10.0)$ & $5.0(1.0-10.0)$ \\
\hline \multicolumn{4}{|l|}{ Baseline BDI-II total } \\
\hline Mean (SD) & $6.0(6.8)$ & $6.4(6.9)$ & $6.2(6.8)$ \\
\hline Median (range) & $4.0(0.0-37.0)$ & $4.0(0.0-43.0)$ & $4.0(0.0-43.0)$ \\
\hline
\end{tabular}

Abbreviations: BPI, Brief Pain Inventory; BDI-II, Beck Depression Inventory-II; CLBP, chronic low back pain; SD, standard deviation. 


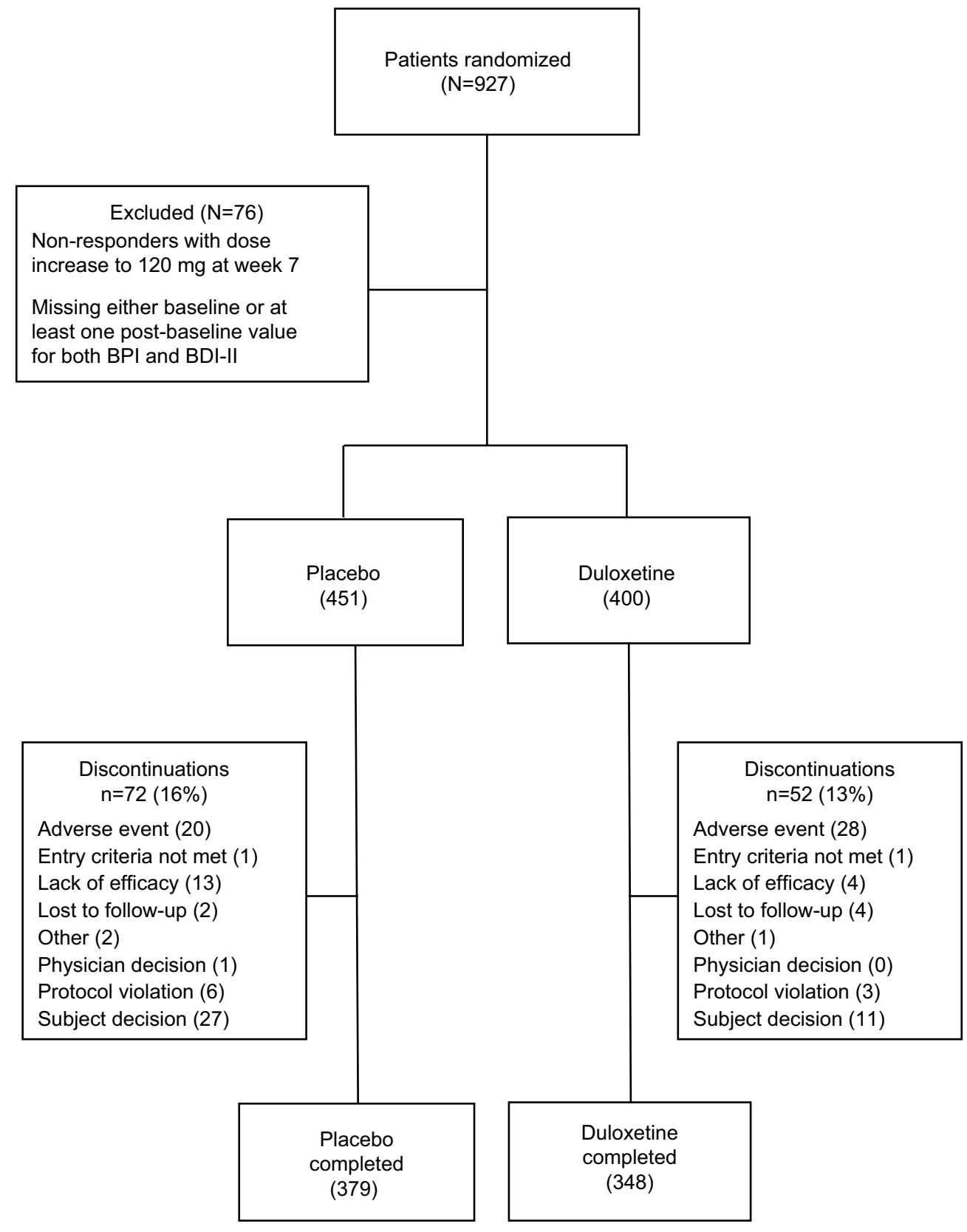

Figure 2 CONSORT diagram illustrating patient disposition.

Abbreviations: BDI-II, Beck Depression Inventory-II; BPI, Brief Pain Inventory.

\section{Subgroup analyses of duloxetine treatment}

\section{Subgroups based on $\mathrm{BPI}<6$ and $\mathrm{BPI} \geq 6$}

The baseline BPI values of the duloxetine and placebo groups of the BPI $<6$ subgroup were (mean \pm SD) $4.4 \pm 0.61$ and $4.4 \pm 0.71$, respectively, and $6.9 \pm 1.03$ and $6.8 \pm 0.98$, respectively for the BPI $\geq 6$ subgroup. For both of these subgroups, the reduction in BPI of duloxetine-treated patients was significantly $(p<0.001)$ greater than that of the placebo group, and appeared to be greater in the BPI $\geq 6$ group (Figure 5A). Duloxetine significantly $(p=0.020)$ reduced

BDI-II scores relative to placebo in the BPI $<6$ subgroup, but not ( $p=0.274$ ) in the BPI $\geq 6$ subgroup (Figure 5B). Path analyses revealed that the direct effect of duloxetine on pain symptoms of the BPI $<6$ and BPI $\geq 6$ subgroups accounted for $88.1 \%$ and $93.4 \%$, respectively, of the total analgesic effect (Figure 5C).

\section{Subgroups based on BDI-II $<6$ and BDI-II $\geq 6$}

Duloxetine treatment produced significantly greater reductions in BPI scores compared with placebo for BDI-II $<6$ and BDI-II $\geq 6$ subgroups (Figure 6A). Duloxetine and placebo 

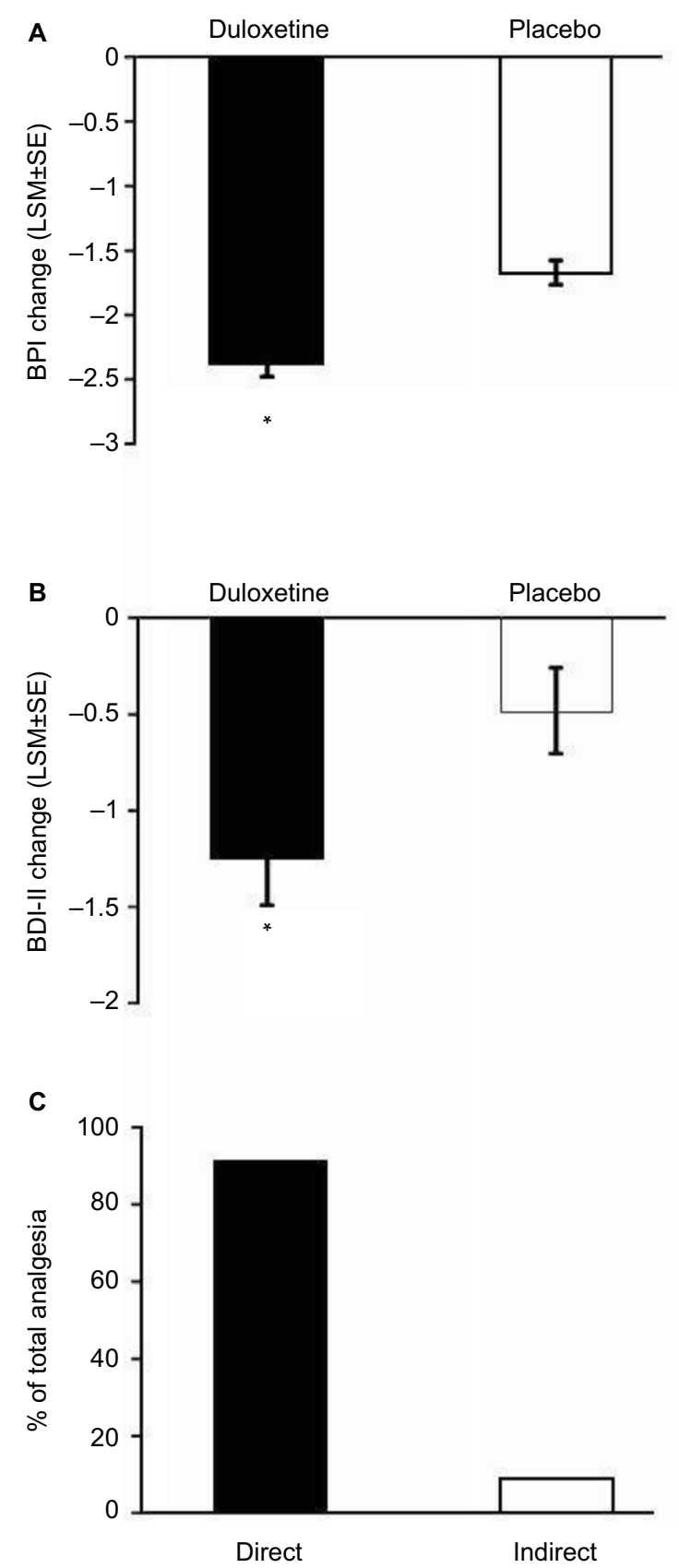

Figure 3 Change at endpoint from baseline of BPI average pain scores for CLBP patients treated with duloxetine and placebo $(\mathbf{A})$. * indicates significant $(p<0.00 \mathrm{l}$, ANCOVA) difference from placebo. Change from baseline of BDI-II total scores for CLBP patients treated with duloxetine and placebo $(B) . *$ indicates significant $(p=0.015, A N C O V A)$ difference from placebo. The error bars on graphs represent SE. Path analyses showing the percent of total analgesic effect attributed to a direct and indirect effect of duloxetine on pain (C).

Abbreviations: ANCOVA, analysis of covariance; BDI-II, Beck Depression Inventory-II; BPI, Brief Pain Inventory; CLBP, chronic low back pain; LSM, least squares mean; SE, standard error.

treatments produced very small nonsignificant increases in BDI-II scores of the BDI-II $<6$ subgroup, whereas duloxetine produced a significantly $(p=0.010)$ greater reduction in BDI-II scores than did placebo in the BDI-II $\geq 6$ subgroup
(Figure 6B). Path analyses revealed that the direct effect of duloxetine on pain symptoms of the BDI-II $<6$ and BDI-II $\geq 6$ subgroups accounted for $98.5 \%$ and $79.0 \%$, respectively, of the total analgesic effect (Figure 6C).

\section{Subgroups based on gender}

Duloxetine produced significantly greater reductions in BPI scores than placebo in both female $(p<0.001)$ and male $(p=0.031)$ patients (Figure 7A). In addition, duloxetine produced a significantly $(p=0.008)$ greater reduction in BDI-II scores than did placebo in female patients and nonsignificant changes in male patients (Figure 7B). Path analyses revealed that the direct effect of duloxetine on pain symptoms was $88.1 \%$ of the total analgesic effect for female patients, and was $97.6 \%$ for male patients (Figure $7 \mathrm{C}$ ).

\section{Discussion}

Duloxetine is a potent and selective serotonin and norepinephrine reuptake inhibitor that acts within the central nervous system and can enhance the action of the endogenous descending inhibitory pain modulatory systems in the lumbar spinal dorsal horn. ${ }^{19,20,27}$ Clinical consequences of this mechanism of action are antidepressant and analgesic effects for chronic pain, and duloxetine has shown clinical analgesic efficacy in diabetic neuropathy, fibromyalgia osteoarthritis of the knee, and CLBP. ${ }^{6,11,17,21}$ The analgesic effect is independent from the antidepressant activity. ${ }^{25}$ The main purpose of the present investigation was to demonstrate this claim in patients with CLBP. These conditions are associated with dysfunction of endogenous pain modulation and associated with diminished conditioned pain modulation, and it is highly likely that duloxetine, by enhancing noradrenergic and serotonergic neurotransmission, engages descending pain modulatory systems, and may normalize this dysfunctional process. ${ }^{28,29}$

Path analyses showed that the majority of the pain reduction in pain scores can be attributed to a direct effect of duloxetine on pain, and only a small proportion due to a reduction of depressive symptoms. The direct effect on pain accounted for $91.1 \%$ of the total analgesic effect of duloxetine. A recent path analysis performed only with data from HMGY in Japan showed a similar result. ${ }^{21}$ In that study, a direct effect of $97.3 \%$ was shown for duloxetine in treating CLBP. ${ }^{21}$ Patients with CLBP treated with $60 \mathrm{mg} /$ day of duloxetine had a significant, progressive improvement in pain scores relative to placebo-treated patients. It should also be noted that over time, the direct effect of duloxetine on pain symptoms diminished slightly and the indirect effect through antidepressant activity increased accordingly. This 

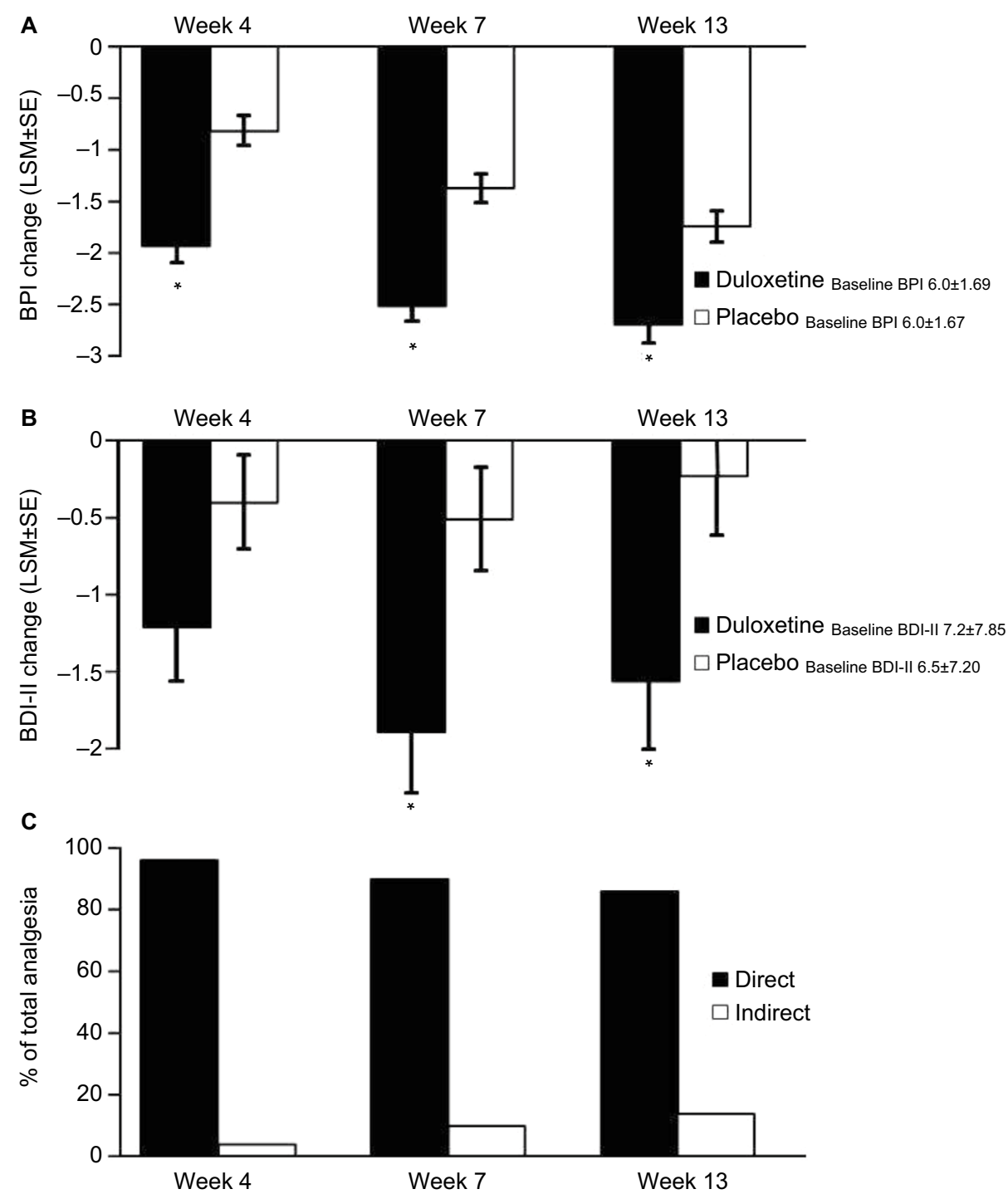

Figure 4 Change from baseline of BPI average pain scores at weeks 4, 7, and 13 for CLBP patients receiving duloxetine or placebo. Changes shown are the difference from the initial baseline BPI value (A). * indicates significant ( $p<0.00 \mathrm{I}$, ANCOVA) difference from placebo. Change from baseline of BDI-II total scores for CLBP patients treated with duloxetine and placebo. Changes shown are the difference from the initial baseline BDI-II value (B). * indicates significant ( $p=0.007$ and 0.022 , ANCOVA, at weeks 7 and 13, respectively) difference from placebo. The mean baseline BPI $( \pm S D)$ and BDI-II $( \pm S D)$ are shown for each group. Error bars on graphs represent SE. Path analyses showing the percent of total analgesic effect attributed to a direct and indirect effect of duloxetine on pain at each time point (C).

Abbreviations: ANCOVA, analysis of covariance; BDI-II, Beck Depression Inventory-II; BPI, Brief Pain Inventory; CLBP, chronic low back pain; LSM, least squares mean; SD standard deviation; SE, standard error.

result is consistent with the observations made recently in patients with MDD and pain symptoms. ${ }^{25}$ In that study, the time-dependent change from a predominance of direct effect to indirect effect of duloxetine on pain symptoms was much more pronounced, since these patients also had MDD and improvement of associated depressive symptoms. ${ }^{25}$

Depression is a common comorbidity in chronic pain states, including CLBP. ${ }^{10,21}$ Severity of pain and its impact on functional impairment are associated with a greater risk of depression, and depression is associated with greater pain complaints, impairment, and poorer treatment outcomes. ${ }^{9}$
Consequently, it is reasonable to expect that alleviation of depressive symptoms could reduce pain symptoms as well. Path analyses recently showed that duloxetine has both a direct and an indirect analgesic effect in patients with MDD and symptoms of physical pain, showing that it is able to produce analgesia independent of an antidepressant effect. ${ }^{25}$ It is very likely that the analgesic effect of duloxetine occurs independently of its antidepressant effect in the present study. This likelihood is especially emphasized since patients with MDD were specifically excluded from the investigation. 

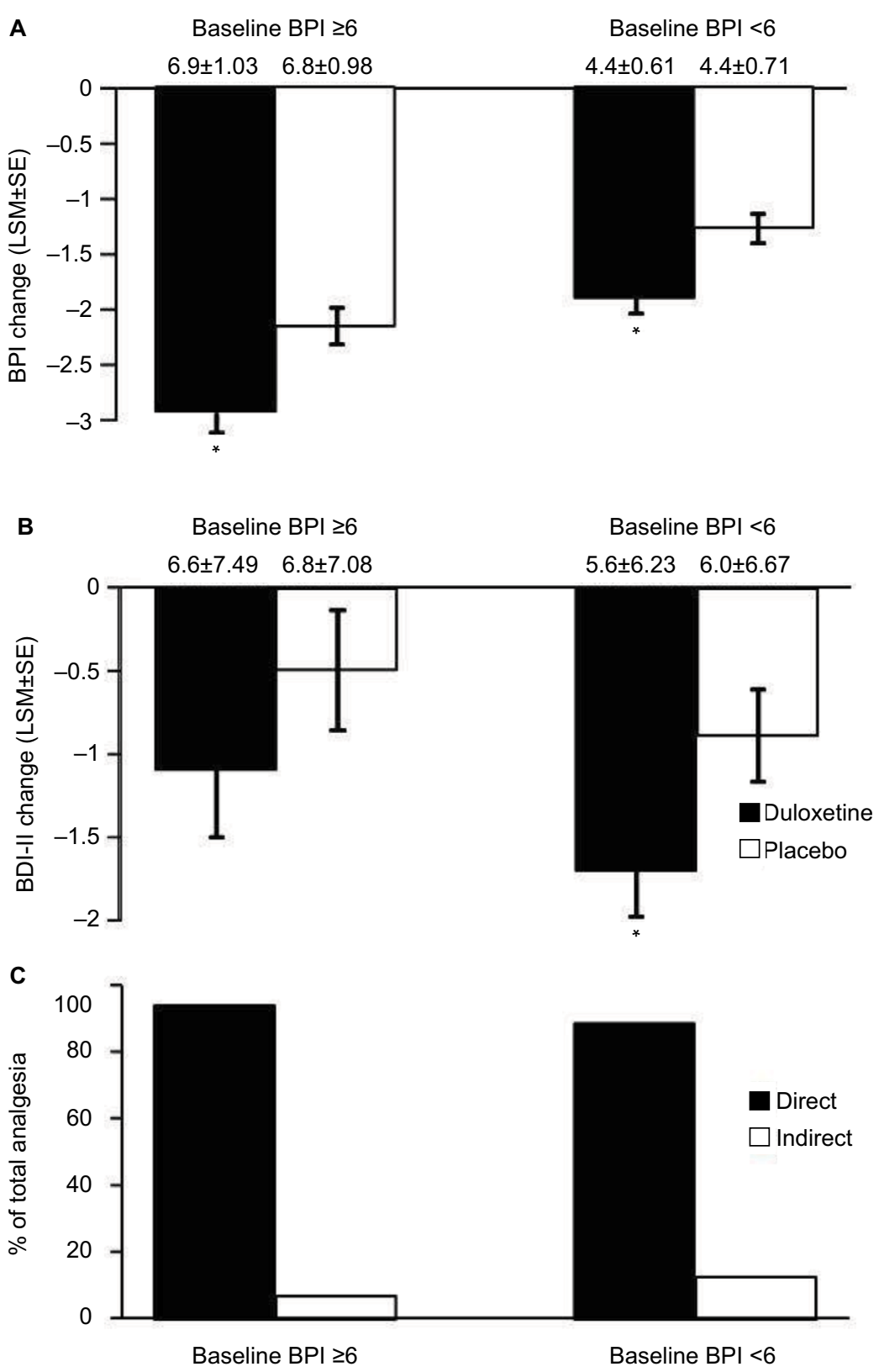

Figure 5 Subgroups formed from baseline BPI $<6(N=464)$ and $B P I$ scores $\geq 6(N=387)$. Endpoint change from baseline of $B P I$ average pain scores for subgroups of $C L B P$ patients receiving duloxetine or placebo $(\mathbf{A}) . *$ indicates significant $(p<0.00 \mathrm{I}$, ANCOVA) difference from placebo. Change from baseline of BDI-II total scores for subgroups of CLBP patients treated with duloxetine and placebo (B). * indicates significant ( $p=0.020$, ANCOVA) difference from placebo. The mean baseline BPI ( \pm SD) and BDI-II $( \pm S D)$ are shown for each subgroup. Error bars on graphs represent SE. Path analyses showing the percent of total analgesic effect attributed to a direct and indirect effect of duloxetine on pain for each subgroup $(\mathbf{C})$.

Abbreviations: ANCOVA, analysis of covariance; BDI-II, Beck Depression Inventory-II; BPI, Brief Pain Inventory; CLBP, chronic low back pain; LSM, least squares mean; SD standard deviation; SE, standard error.

In the present analysis, the BDI-II $\geq 6$ subgroup still had relatively low mean scores, of $\sim 12$, reflecting the fact that none of the included patients were diagnosed with depressive disorders. Consequently, it is not surprising that there was no significant difference in the direct effect of duloxetine between the 2 groups. Thus, the results of the path analyses, together with the disconnect between changes in depressive symptoms and pain symptoms, and the absence of patients with MDD all support that duloxetine has a direct effect on pain relief, independent of its antidepressant activity.

Parsing subgroups based on baseline BPI scores, BDI-II scores, and gender did not appear to lead to any substantial changes in the percentage of analgesia attributed to the direct effect of duloxetine. Patients with a higher baseline BPI score (ie, $\geq 6$ ) showed a markedly greater reduction in BPI than 

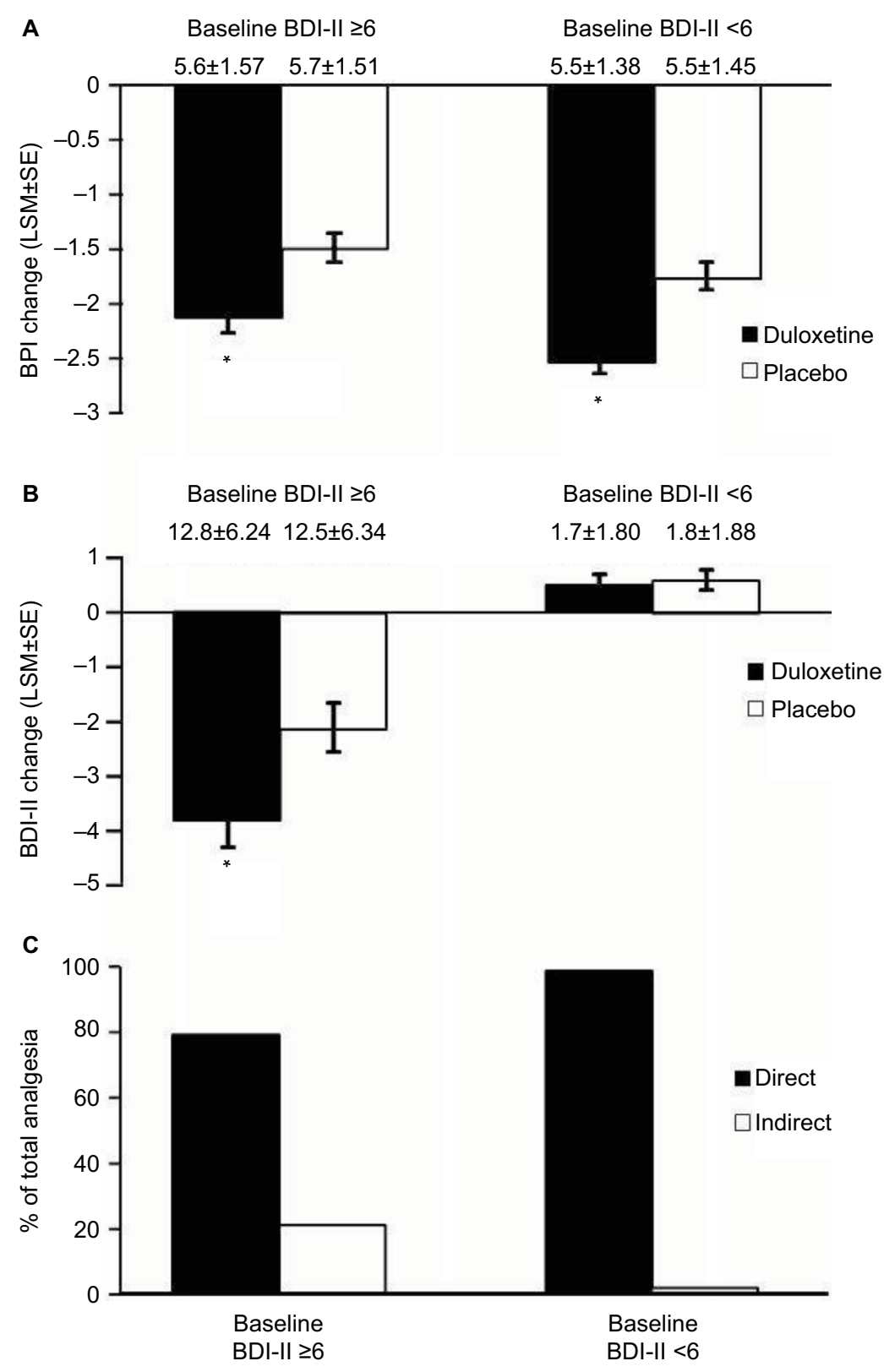

Figure 6 Subgroups formed from baseline BDI-II $<6(\mathrm{~N}=50 \mathrm{I})$ and $\mathrm{BDI}-\mathrm{Il}$ scores $\geq 6(\mathrm{~N}=350)$. Endpoint change from baseline of $\mathrm{BPI}$ scores for both subgroups of $\mathrm{CLBP}$ patients receiving duloxetine or placebo $(\mathbf{A})$. * indicates significant ( $p<0.00$, ANCOVA, for BDI-II $<6 ; p=0.003$ for BDI-II $\geq 6)$ difference from placebo. Endpoint change from baseline of BDI-II scores for both subgroups of CLBP patients treated with duloxetine and placebo $(B) . *$ indicates significant $(p=0.010$, ANCOVA) difference from placebo. Error bars on graphs represent SE. The mean baseline BPI $( \pm S D)$ and BDI-II $( \pm S D)$ are shown for each group. Path analyses showing the percent of total analgesic effect attributed to a direct and indirect effect of duloxetine on pain for each subgroup (C). * indicates significant $(p \leq 0.05$, ANCOVA) difference from placebo.

Abbreviations: ANCOVA, analysis of covariance; BDI-II, Beck Depression Inventory-II; BPI, Brief Pain Inventory; CLBP, chronic low back pain; LSM, least squares mean; SD standard deviation; SE, standard error.

did those with lower baseline pain scores. This observation is consistent with a greater range over which duloxetine could exert an analgesic effect. The same group, however, showed less reduction in depressive symptoms, underscoring a possible lack of association between pain relief and antidepressive activity. Baseline depressive scores did not appear to affect the analgesic effect of duloxetine, as both groups showed an approximately similar degree of analgesia. Patients with high baseline BDI-II scores showed marked reduction in these scores with duloxetine, underscoring its antidepressant activity. This group showed the lowest attribution (\%) of direct effects to duloxetine, and a somewhat higher indirect effect relative to all of the other subgroups, which is also consistent with the antidepressant effect of duloxetine. Males and females showed similar levels of pain relief and of the direct effect (\%) on analgesia, although female patients appeared to show a somewhat greater level of antidepressant activity as well. 
A
Female
Male

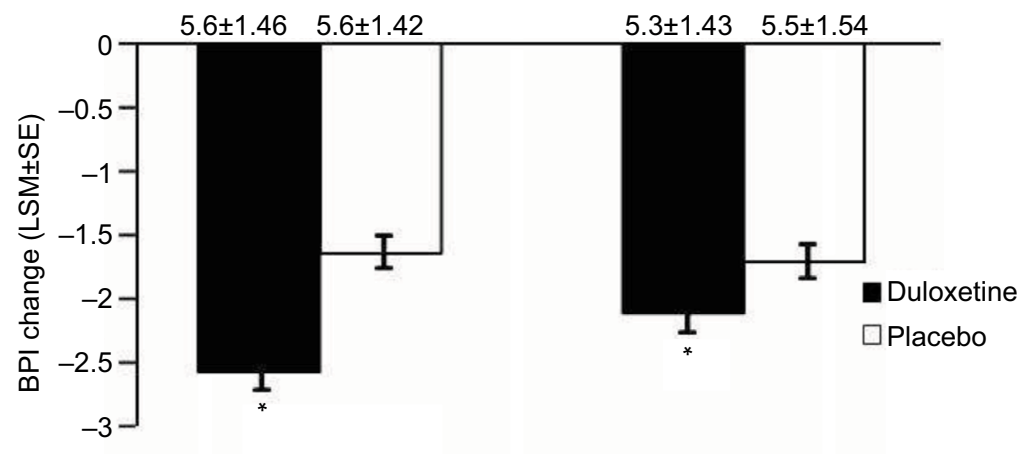

B

Female

Male
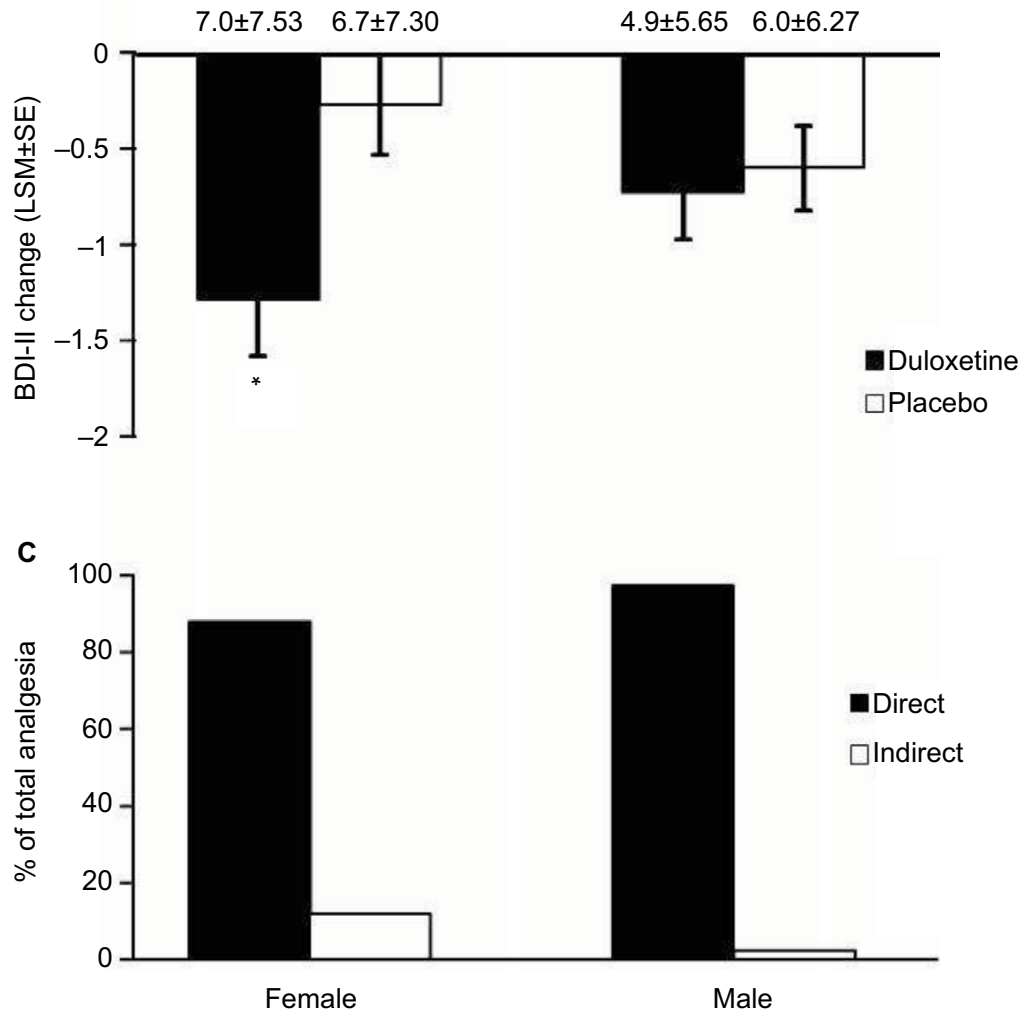

Figure 7 Subgroups formed from male ( $N=379)$ and female $(\mathrm{N}=472)$ CLBP patients. Endpoint change from baseline of $B P I$ scores for female and male $C L B P$ patients receiving duloxetine or placebo $(\mathbf{A})$. * indicates significant $(p<0.00 \mathrm{I}$, ANCOVA for females, $p=0.03 \mathrm{I}$ for males) difference from placebo. Endpoint change from baseline of BDI-II scores for female and male CLBP patients treated with duloxetine and placebo $(B) . *$ indicates significant $(p=0.008, A N C O V A)$ difference from placebo. The mean baseline BPI $( \pm S D)$ and $\mathrm{BDI}-\mathrm{II}( \pm \mathrm{SD})$ are shown for each group. Error bars on graphs represent SE. Path analyses showing the percent of total analgesic effect attributed to a direct and indirect effect of duloxetine on pain for female and male CLBP patients $(\mathbf{C}) . *$ indicates significant $(p \leq 0.05$, ANCOVA) difference from placebo.

Abbreviations: ANCOVA, analysis of covariance; BDI-II, Beck Depression Inventory-II; BPI, Brief Pain Inventory; CLBP, chronic low back pain; LSM, least squares mean; SD standard deviation; SE, standard error.

The results of this investigation strongly suggest that a substantial part of pain relief due to duloxetine is largely due to a direct effect. This is consistent with duloxetine's ability to enhance endogenous pain modulatory systems, which is an effect independent of its antidepressant activity.

A limitation of the present investigation is that the path analysis was based on a hypothesis that a factor that induces pain relief is the antidepressive effect of duloxetine. Even though the depressive symptom is one of the emotional components, the present study focused on the correlations between pain and depressive symptoms. Another limitation is that the present investigation is limited to patients with low depressive symptoms. Patients with MDD were excluded.

\section{Acknowledgments}

The authors thank Noelle Gasco, full-time employee of inVentiv Health Clinical, LLC, for editorial support. These trials and the preparation of the manuscript were sponsored 
by Eli Lilly and Company, Indianapolis, IN, USA. Eli Lilly and Company contracted inVentiv Health Clinical for writing and editorial support.

\section{Disclosure}

HE, SF, JF, NS, and LA are full-time employees of Eli Lilly Japan K.K. TT is a full-time employee and minor stock holder of Shionogi \& Co. Ltd. TU has received honoraria from Hisamitsu, Pfizer, Shionogi, Lilly, and Daiichi-Sankyo and grant/research funding from Seikagaku Corporation, Nihon Zoki, Astellas, Pfizer, Eisai, Daiichi-Sankyo, Medtronic, and Kyowa-Hakko Kirin. MHO is a full-time employee of inVentiv Health Clinical, LLC. The authors report no other conflicts of interest in this work.

\section{References}

1. Sadosky AB, DiBonaventura M, Cappelleri JC, Ebata N, Fujii K. The association between lower back pain and health status, work productivity, and health care resource use in Japan. J Pain Res. 2015;8: 119-130.

2. Deyo RA, Weinstein JN. Low back pain. $N$ Engl J Med. 2001;344(5): 363-370.

3. Fujii T, Matsudaira K. Prevalence of low back pain and factors associated with chronic disabling back pain in Japan. Eur Spine J. 2013;22(2):432-438.

4. Andersson GB. Epidemiological features of chronic low-back pain. Lancet. 1999;354(9178):581-585.

5. Meucci RD, Fassa AG, Faria NM. Prevalence of chronic low back pain: systematic review. Rev Saude Publica. 2015;49:73-82.

6. Skljarevski V, Zhang S, Desaiah D, et al. Duloxetine versus placebo in patients with chronic low back pain: a 12-week, fixed-dose, randomized, double-blind trial. J Pain. 2010;11(12):1282-1290.

7. Kelly GA, Blake C, Power CK, O'keeffe D, Fullen BM. The association between chronic low back pain and sleep: a systematic review. Clin J Pain. 2011;27(2):169-181.

8. Reme SE, Tangen T, Moe T, Eriksen HR. Prevalence of psychiatric disorders in sick listed chronic low back pain patients. Eur J Pain. 2011;15(10):1075-1080.

9. Bair MJ, Robinson RL, Katon W, Kroenke K. Depression and pain comorbidity: a literature review. Arch Intern Med. 2003;163(20): 2433-2445.

10. Polatin PB, Kinney RK, Gatchel RJ, Lillo E, Mayer TG. Psychiatric illness and chronic low-back pain. The mind and the spine - which goes first? Spine (Phila Pa 1976). 1993;18(1):66-71.

11. Skljarevski V, Ossanna M, Liu-Seifert H, et al. A double-blind, randomized trial of duloxetine versus placebo in the management of chronic low back pain. Eur J Neurol. 2009;16(9):1041-1048.

12. Chou R. In the clinic. Low back pain. Ann Intern Med. 2014;160(11): ITC6-1.

13. Hoy D, Brooks P, Blyth F, Buchbinder R. The epidemiology of low back pain. Best Pract Res Clin Rheumatol. 2010;24(6):769-781.
14. Clauw DJ. Diagnosing and treating chronic musculoskeletal pain based on the underlying mechanism(s). Best Pract Res Clin Rheumatol. 2015;29(1):6-19.

15. Corrêa JB, Costa LO, de Oliveira NT, Sluka KA, Liebano RE. Central sensitization and changes in conditioned pain modulation in people with chronic nonspecific low back pain: a case-control study. Exp Brain Res. 2015;233(8):2391-2399.

16. Giesecke T, Gracely RH, Grant MA, et al. Evidence of augmented central pain processing in idiopathic chronic low back pain. Arthritis Rheum. 2004;50(2):613-623.

17. Skljarevski V, Desaiah D, Liu-Seifert H, et al. Efficacy and safety of duloxetine in patients with chronic low back pain. Spine (Phila Pa 1976). 2010;35(13):E578-E585.

18. Webster LR, Markman J. Medical management of chronic low back pain: efficacy and outcomes. Neuromodulation. 2014;17(Suppl 2):18-23.

19. Jones CK, Peters SC, Shannon HE. Efficacy of duloxetine, a potent and balanced serotonergic and noradrenergic reuptake inhibitor, in inflammatory and acute pain models in rodents. J Pharmacol Exp Ther. 2005;312(2):726-732.

20. Koch S, Hemrick-Luecke SK, Thompson LK, et al. Comparison of effects of dual transporter inhibitors on monoamine transporters and extracellular levels in rats. Neuropharmacology. 2003;45(7):935-944.

21. Konno S, Oda N, Ochiai T, Alev L. A randomized, double-blind, placebocontrolled phase III trial of duloxetine monotherapy in Japanese patients with chronic low back pain. Spine (Phila Pa 1976). Epub 2016 May 23.

22. Brecht S, Courtecuisse C, Debieuvre C, et al. Efficacy and safety of duloxetine $60 \mathrm{mg}$ once daily in the treatment of pain in patients with major depressive disorder and at least moderate pain of unknown etiology: a randomized controlled trial. J Clin Psychiatry. 2007;68(11):1707-1716.

23. Gaynor PJ, Gopal M, Zheng W, Martinez JM, Robinson MJ, Marangell LB. A randomized placebo-controlled trial of duloxetine in patients with major depressive disorder and associated painful physical symptoms. Curr Med Res Opin. 2011;27(10):1849-1858.

24. Schneider E, Linden M, Weigmann H, et al. Early reduction in painful physical symptoms is associated with improvements in long-term depression outcomes in patients treated with duloxetine. BMC Psychiatry. $2011 ; 11: 150$.

25. Schwab J, Bialow M, Clemmons R, Martin P, Holzer C. The Beck depression inventory with medical inpatients. Acta Psychiatr Scand. 1967;43(3):255-266.

26. Harada E, Tokuoka H, Fujikoshi S, et al. Is duloxetine's effect on painful physical symptoms in depression an indirect result of improvement of depressive symptoms? Pooled analyses of three randomized controlled trials. Pain. 2016;157(3):577-584.

27. Robinson MJ, Sheehan D, Gaynor PJ, et al. Relationship between major depressive disorder and associated painful physical symptoms: analysis of data from two pooled placebo-controlled, randomized studies of duloxetine. Int Clin Psychopharmacol. 2013;28(6):330-338.

28. Hoshino H, Obata H, Saito S. Antihyperalgesic effect of duloxetine and amitriptyline in rats after peripheral nerve injury: influence of descending noradrenergic plasticity. Neurosci Lett. 2015;602:62-67.

29. Staud R. Abnormal endogenous pain modulation is a shared characteristic of many chronic pain conditions. Exp Rev Neurother. 2012;12(5):577-585.

30. Ossipov MH, Morimura K, Porreca F. Descending pain modulation and chronification of pain. Curr Opin Support Palliat Care. 2014;8(2): 143-151. 
The Journal of Pain Research is an international, peer reviewed, open access, online journal that welcomes laboratory and clinical findings in the fields of pain research and the prevention and management of pain. Original research, reviews, symposium reports, hypothesis formation and commentaries are all considered for publication.
The manuscript management system is completely online and includes a very quick and fair peer-review system, which is all easy to use. Visit http://www.dovepress.com/testimonials.php to read real quotes from published authors. 